\title{
Analysis of Switchable Spin Torque Oscillator for Microwave Assisted Magnetic Recording
}

\author{
Mingsheng Zhang, Tiejun Zhou, and Zhimin Yuan \\ Data Storage Institute, Agency for Science, Technology and Research ( ${ }^{*}$ STAR), Singapore 117608 \\ Correspondence should be addressed to Tiejun Zhou; zhou_tiejun@dsi.a-star.edu.sg
}

Received 10 November 2014; Revised 25 February 2015; Accepted 1 March 2015

Academic Editor: Joseph S. Poon

Copyright ( 2015 Mingsheng Zhang et al. This is an open access article distributed under the Creative Commons Attribution License, which permits unrestricted use, distribution, and reproduction in any medium, provided the original work is properly cited.

\begin{abstract}
A switchable spin torque oscillator (STO) with a negative magnetic anisotropy oscillation layer for microwave assisted magnetic recording is analyzed theoretically and numerically. The equations for finding the STO frequency and oscillation angle are derived from Landau-Lifshitz-Gilbert (LLG) equation with the spin torque term in spherical coordinates. The theoretical analysis shows that the STO oscillating frequency remains the same and oscillation direction reverses after the switching of the magnetization of the spin polarization layer under applied alternative magnetic field. Numerical analysis based on the derived equations shows that the oscillation angle increases with the increase of the negative anisotropy energy density (absolute value) but decreases with the increase of spin current, the polarization of conduction electrons, the saturation magnetization, and the total applied magnetic field in the $z$ direction. The STO frequency increases with the increase of spin current, the polarization of conduction electrons, and the negative anisotropy energy density (absolute value) but decreases with the increase of the saturation magnetization and the total applied magnetic field in the $z$ direction.
\end{abstract}

\section{Introduction}

Microwave assisted magnetic recording (MAMR) is one potential technology to overcome the superparamagnetic effect of perpendicular magnetic recording in the hard disk drive. A microwave field matching with the ferromagnetic resonance of recording media excites a large angle precession of magnetization, resulting in a significant reduction in switching field. Using microwave-assisted magnetic switching, it is possible to write data into high magnetocrystalline anisotropy recording media, such as $\mathrm{FePt}$ and $\mathrm{CoPt}$, which have sufficient thermal stability at very small grain size.

The angular momentum carried by the spin-polarized current applies a torque on the magnetization vector leading to either precession or reversal through spin-transfertorque effect $[1,2]$. The current-induced magnetization precession enables magnetic nanostructure to be a tunable high-frequency spin-torque oscillator (STO) [3]. The highfrequency magnetization precession in STO can generate localized microwave suitable for the application for MAMR, as proposed in $[4,5]$. Furthermore, the fabrication processes of STO are compatible with current thin film perpendicular magnetic recording head and are easy to integrate with the current recording technology.

For the real application of STO for MAMR, the STO should be near the writing pole to avoid field decay with the distance away from STO, as shown in the thin film magnetic head in Figure 1. The STO basically consists of a spin polarization layer, a spacer, and an oscillation layer. The STO is located between the writing pole and trailing shield. The microwave generated by the STO can assist the magnetic field from the writing pole to switch the media.

There is very strong magnetic field in the gap between the writing pole and trailing shield; the STO with the negative magnetic anisotropy oscillation layer can oscillate stably under the very wide range of applied fields and injected spin currents [6]. Therefore, STO with negative magnetic anisotropy oscillation layer is preferred. The oscillation frequency and oscillation angle of the switchable STO, which, together with the saturation magnetization of oscillation layer, determine the microwave frequency and the amplitude 


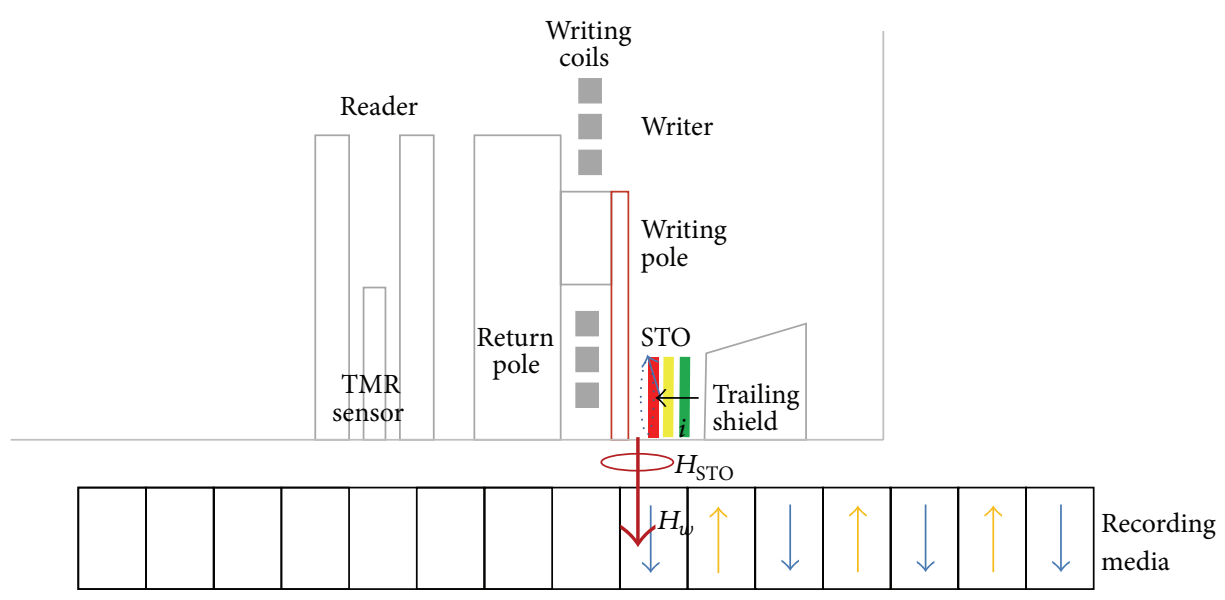

FIGURE 1: Spin torque oscillator in thin film magnetic head.

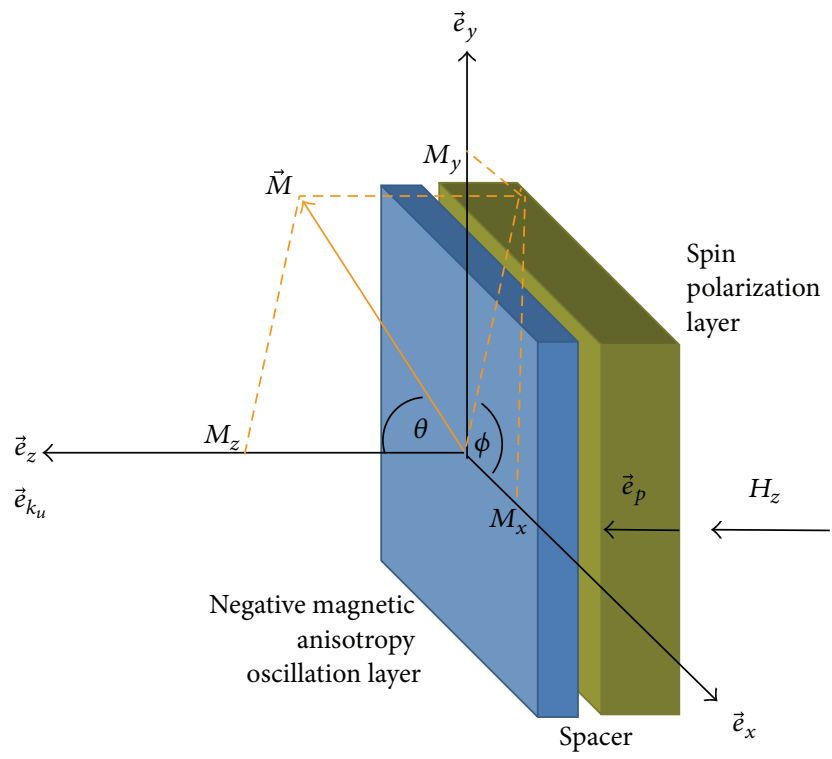

FIGURE 2: Spin torque oscillator with a negative magnetic anisotropy oscillation layer.

(critical for MAMR application), are investigated in detail in this paper.

\section{Theoretical Analysis of the Switchable Spin Torque Oscillator}

2.1. Theoretical Analysis of the STO Frequency and Oscillation Angle. The basic structure of the STO and the coordinates used for the analysis in this paper are shown in Figure 2. A simple approach to describe current induced magnetization oscillation of the oscillation layer is to fix the magnetization of spin polarization layer and consider the oscillation layer magnetization as a uniform macrospin. The dynamics of the oscillation layer magnetization follows the LandauLifshitz-Gilbert (LLG) equation with the Slonczewski's spin torque term:

$$
\begin{aligned}
\frac{d \vec{M}}{d t}= & -\gamma_{0}\left(\vec{M} \times \vec{H}_{\mathrm{eff}}\right)+\frac{\alpha}{M_{0}} \vec{M} \times \frac{d \vec{M}}{d t} \\
& +\frac{\gamma_{0} I_{s} \hbar G(\psi)}{\mu_{0} V M_{0}^{2} 2 e} \vec{M} \times\left(\vec{M} \times \vec{e}_{p}\right),
\end{aligned}
$$

where $\gamma_{0}$ is the gyromagnetic factor, $\vec{H}_{\mathrm{eff}}$ is the effective magnetic field, $\alpha$ is the damping constant, $M_{0}$ is saturation magnetization, $I_{s}$ is the current passing through the STO, $\hbar$ is the reduced Planck constant, $\mu_{0}$ is the permeability of free space, $V$ is the volume of the oscillation layer, $e$ is the charge of an electron $\left(-1.60 \times 10^{-19} \mathrm{C}\right), \vec{e}_{p}$ is current polarization, and $G(\psi)$ is the spin transfer efficiency function given by $G(\psi)=\left[-4+(1+P)^{3} \cdot\left(\left(3+\vec{e}_{p} \cdot \vec{m}\right) / 4 P^{3 / 2}\right)\right]^{-1}$, where $\vec{m}=\vec{M} / M_{s}$ and $P$ is the polarization of conduction electrons.

If the spin torque term is included into the effective magnetic field, (1) can be rewritten as

$$
\frac{d \vec{M}}{d t}=-\gamma_{0} \vec{M} \times \vec{H}_{\mathrm{eff}}^{A}+\frac{\alpha}{M_{s}} \vec{M} \times \frac{d \vec{M}}{d t},
$$

where $\vec{H}_{\text {eff }}^{A}=\vec{H}_{\text {eff }}+\left(I_{s} \hbar G(\psi) / \mu_{0} V M_{s}^{2} 2 e\right)\left(\vec{M} \times \vec{e}_{p}\right)$, and (2) is the same as the traditional LLG equation in format.

The LLG equation given in the spherical coordinates can be expressed as

$$
\begin{aligned}
& \frac{d \theta}{d t}=\frac{\gamma_{0}}{1+\alpha^{2}}\left[h_{\mathrm{eff} \phi}^{A}+\alpha h_{\mathrm{eff} \theta}^{A}\right], \\
& \frac{d \phi}{d t}=\frac{\gamma_{0}}{1+\alpha^{2}} \frac{1}{\sin \theta}\left[\alpha h_{\mathrm{eff} \phi}^{A}-h_{\mathrm{eff} \theta}^{A}\right],
\end{aligned}
$$

where $h_{\mathrm{eff} \phi}^{A}$ and $h_{\mathrm{eff} \theta}^{A}$ are the normalized total effective field along $\vec{e}_{\theta}$ and $\vec{e}_{\phi}$ in the spherical coordinates.

Here the following conditions are assumed: (1) the uniaxial magnetic anisotropy of the spin polarization layer is 
along the $z$ axis, (2) magnetization of the spin polarization layer is fixed along the $+z$ axis, (3) the dimensions of STO in the $x y$ direction are the same, and (4) the magnetic field is only applied along the $z$ axis. The effective magnetic field is calculated by the energy variation with magnetization; the $h_{\mathrm{eff} \phi}^{A}$ and $h_{\mathrm{eff \theta}}^{A}$ can be expressed as

$$
\begin{aligned}
& h_{\mathrm{eff} \theta}=-\frac{H_{a z}}{M_{0}} \sin \theta-\frac{K_{u}}{\mu_{0} M_{0}^{2}} \sin 2 \theta-\frac{1}{2}\left(N_{x}-N_{z}\right) \sin 2 \theta, \\
& h_{\mathrm{eff} \phi}=-\frac{I_{s} \hbar G(\theta)}{2 e \mu_{0} V M_{0}^{2}} \sin \theta,
\end{aligned}
$$

where $H_{a z}$ is total applied magnetic field on the oscillation layer that includes the external applied field and the demagnetizing field from the spin polarization layer. $K_{u}$ is the anisotropy energy density, and $N_{x}, N_{z}$ are the demagnetizing factors of the oscillation layer. Thus (3a) and (3b) can be expressed as

$$
\begin{array}{r}
\frac{d \theta}{d t}=-\frac{\gamma_{0} \sin \theta}{1+\alpha^{2}}\left\{\frac{I_{s} \hbar G(\theta)}{2 e \mu_{0} V M_{0}^{2}}+\alpha\left[\frac{H_{a z}}{M_{0}}+\frac{2 K_{u}}{\mu_{0} M_{0}^{2}} \cos \theta\right.\right. \\
\left.\left.+\left(N_{x}-N_{z}\right) \cos \theta\right]\right\},
\end{array}
$$

$$
\begin{gathered}
\frac{d \phi}{d t}=\frac{\gamma_{0}}{1+\alpha^{2}}\left[-\frac{\alpha I_{s} \hbar G(\theta)}{2 e \mu_{0} V M_{0}^{2}}+\frac{H_{a z}}{M_{0}}+\frac{2 K_{u}}{\mu_{0} M_{0}^{2}} \cos \theta\right. \\
\left.+\left(N_{x}-N_{z}\right) \cos \theta\right] .
\end{gathered}
$$

When $d \theta / d t=-\left(\gamma_{0} \sin \theta /\left(1+\alpha^{2}\right)\right)\left\{I_{s} \hbar G(\theta) / 2 e \mu_{0} V M_{0}^{2}+\right.$ $\left.\alpha\left[H_{a z} / M_{0}+\left(2 K_{u} / \mu_{0} M_{0}^{2}\right) \cos \theta+\left(N_{x}-N_{z}\right) \cos \theta\right]\right\}=0$, we have

$$
\begin{aligned}
H_{a z} & +\left[\frac{2 K_{u}}{\mu_{0} M_{0}}+M_{0}\left(N_{x}-N_{z}\right)\right] \cos \theta \\
& =-\frac{I_{s} \hbar}{2 \alpha e \mu_{0} V M_{0}}\left[-4+(1+P)^{3} \cdot \frac{3+\cos \theta}{4 P^{3 / 2}}\right]^{-1} .
\end{aligned}
$$

The oscillation frequency of the STO can be expressed as

$$
\begin{aligned}
& \omega=\frac{d \phi}{d t}=-\frac{\gamma_{0} \hbar}{2 e \mu_{0}} \frac{I_{s} G\left(\theta_{0}\right)}{\alpha V M_{0}} \text { or } \\
& \omega=\frac{d \phi}{d t}=\gamma_{0}\left[\frac{H_{a z}}{M_{0}}+\frac{2 K_{u}}{\mu_{0} M_{0}^{2}} \cos \theta_{0}+\left(N_{x}-N_{z}\right) \cos \theta_{0}\right],
\end{aligned}
$$

where the angle $\theta_{0}$ is the solution of (6); we define $A=$ $(1+P)^{3} / 4 P^{3 / 2}, B=-4+3 A, \chi=\hbar / 2 \alpha e \mu_{0} V M_{0}, H_{k}=$ $2 K_{u} / \mu_{0} M_{0}+M_{0}\left(N_{x}-N_{z}\right)$, and (6) can be expressed as

$$
\left[\cos \theta+\frac{A}{B}\right]\left[\cos \theta+\frac{H_{a z}}{H_{k}}\right]+\frac{\chi I_{s}}{B H_{k}}=0 .
$$

It is not difficult to find the solution of (8) as

$$
\begin{aligned}
\cos \theta_{0}= & \left(-\left(A H_{k}+B H_{a z}\right)\right. \\
& \left. \pm \sqrt{\left(A H_{k}+B H_{a z}\right)^{2}-4 B H_{k}\left(A H_{a z}+\chi I_{s}\right)}\right) \\
& \cdot\left(2 B H_{k}\right)^{-1} .
\end{aligned}
$$

For the stable oscillation, the solution of $\cos \theta_{0}$ is valid only when it is between -1 and +1 . Inputting the angle $\theta_{0}$ into (7a) or (7b), the oscillation frequency of the STO can be found.

2.2. STO Frequency and Oscillation Angle after Switch. If the external magnetic field along the $z$ axis is strong enough, when its direction is changed from $+z$ to $-z$, the magnetization of spin polarization layer will also change from $+z$ to $-z$ (switchable). The demagnetizing field of spin polarization layer also reverses its direction. Therefore, $H_{a z}$ becomes $-H_{a z}$. The reversal of the spin polarization layer magnetization also causes the current polarization to be reversed. Equation (6) becomes

$$
\begin{aligned}
& -H_{a z}+\left[\frac{2 K_{u}}{\mu_{0} M_{0}}+M_{0}\left(N_{x}-N_{z}\right)\right] \cos \theta \\
& =\frac{I_{s} \hbar}{2 \alpha e \mu_{0} V M_{0}}\left[-4+(1+P)^{3} \cdot \frac{3-\cos \theta}{4 P^{3 / 2}}\right]^{-1} .
\end{aligned}
$$

Equation (10) can be rewritten as

$$
\begin{aligned}
H_{a z} & +\left[\frac{2 K_{u}}{\mu_{0} M_{0}}+M_{0}\left(N_{x}-N_{z}\right)\right](-\cos \theta) \\
& =-\frac{I_{s} \hbar}{2 \alpha e \mu_{0} V M_{0}}\left[-4+(1+P)^{3} \cdot \frac{3+(-\cos \theta)}{4 P^{3 / 2}}\right]^{-1} .
\end{aligned}
$$

It is obvious that the solution of (10) is

$$
\cos \theta_{0}^{\prime}=-\cos \theta_{0} \text {. }
$$

Thus $\theta_{0}^{\prime}$ is equal to $\left(\pi-\theta_{0}\right)$, and the corresponding oscillating frequency is

$$
\begin{aligned}
\omega^{\prime} & =\gamma_{0}\left\{-\frac{H_{a z}}{M_{0}}+\left[\frac{2 K_{u}}{\mu_{0} M_{0}^{2}}+\left(N_{x}-N_{z}\right)\right] \cos \theta_{0}^{\prime}\right\} \\
& =-\gamma_{0}\left\{\frac{H_{a z}}{M_{0}}+\left[\frac{2 K_{u}}{\mu_{0} M_{0}^{2}}+\left(N_{x}-N_{z}\right)\right] \cos \theta_{0}\right\}=-\omega_{0} .
\end{aligned}
$$

Therefore, the oscillating frequency remains the same, but the oscillating direction reverses after the switching of external magnetic field and the magnetization of spin polarization layer.

In the application of STO for MAMR, the magnetic field from writing pole is opposite when 0 or 1 is written. 
The reverse of the oscillating direction and the unchanged frequency match the needs for the microwave-assisted magnetic switching when 0 or 1 is written. If the STO is not switchable, the external magnetic field applied to STO is different for writing of 0 and 1 , which causes a shift of STO oscillation frequency (as shown in the next paragraph) and a mismatch between the STO frequency and the recording media switching frequency, resulting in write-in failures which are the main source of MAMR noise.

For the STO studied in this paper, there is no pinning layer in the polarization layer. However, there is a very strong magnetic field of 5000-8000 Oe along the $\pm z$ direction between the main pole and the trailing shield, where the spin torque oscillator (STO) is placed (Figure 1). This field acts on STO, which makes the polarization layer robust enough against other influential forces such as the dipole field from the oscillation layer (which is 100-500 Oe depending on the thickness and $M_{s}$ of free layer) or the field from magnetic recording grain (which is about 200-400 Oe at a flying height of $3-5 \mathrm{~nm}$ ) or the spin torque it experiences when passing through a current (the equivalent spin torque field is about 100-200 Oe).

\section{Numerical Analysis of STO Frequency and Oscillation Angle}

For microwave assisted magnetic recording (MAMR), STO generates microwave, which is used to reduce the switching field of recording media during writing process. In order to sufficiently reduce the media switching field, the microwave frequency should be tuneable to match the natural precession frequency of the media magnetization and the oscillating amplitude of microwave (i.e., AC magnetic field) should be large enough (about $10 \%$ of the media $H_{k}$ ). Therefore, the microwave frequency and the AC magnetic field are two key parameters for MAMR. In our STO design, the AC magnetic field strength is determined by the STO oscillation angle. Therefore, the discussion on the STO oscillation angle is critical for the application of STO in microwave assisted magnetic recording. Based on the equations above, the relationship between STO oscillation angle/frequency and the relative parameters is numerically analysed below. The dimension of the oscillation layer of STO is $40 \mathrm{~nm} \times 40 \mathrm{~nm} \times 10 \mathrm{~nm}$.

3.1. Injected Spin Current. In our simulation we assume that the saturation magnetization $M_{0}$ is $800 \mathrm{kA} / \mathrm{m}$, the anisotropy energy density $K_{u}$ is $-8 \times 10^{5} \mathrm{~J} / \mathrm{m}^{3}$, and the damping constant $\alpha$ is 0.02 for the oscillation layer. The applied magnetic field (including the demagnetizing field from spin polarization layer) $H_{a z}$ is $10000 \mathrm{Oe}$, and the polarization of the conduction electrons $P$ is 0.35 [7]. We vary the current density $J$ from 0 to $1.25 \times 10^{8} \mathrm{~A} / \mathrm{cm}^{2}$. The numerically calculated results of $\cos \theta$ and frequency are shown in Figure 3. The increase of the spin current results in a decrease in the oscillation angle and an increase in the oscillation frequency. This trend is easily understandable because the larger current injects more spin torque to the oscillation layer and makes the oscillation layer oscillate faster.
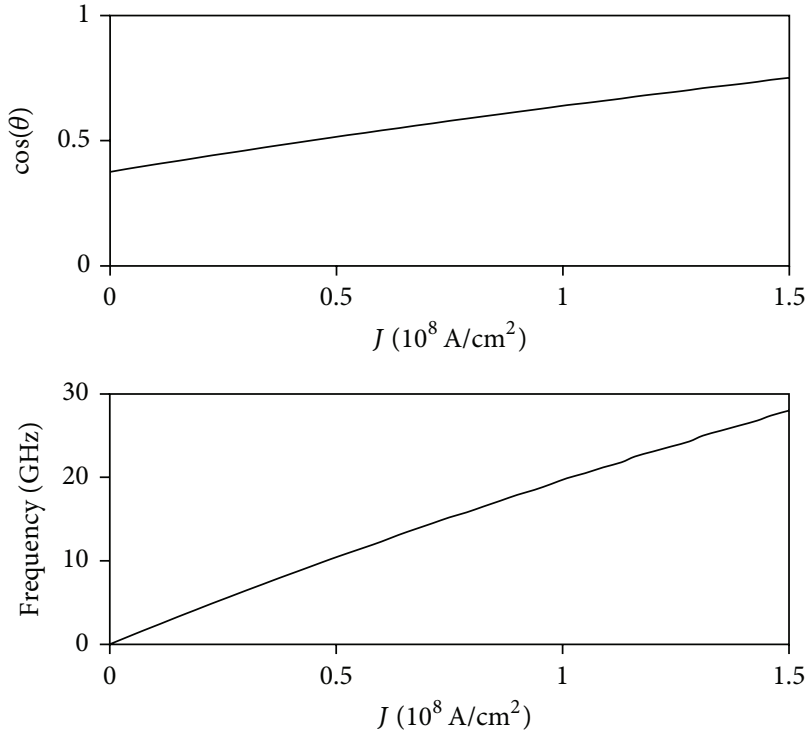

FIGURE 3: Effects of current on STO.
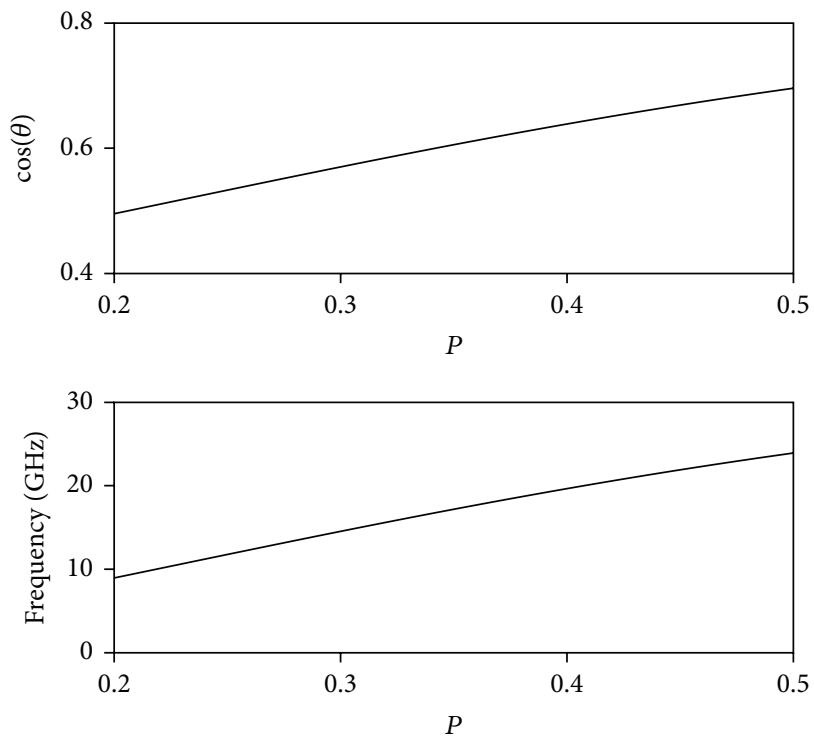

FIGURE 4: Effects of polarization of conduction electrons on STO.

3.2. Polarization of the Conduction Electrons. The simulation parameters are the same as those in Section 3.1, except for a fixed current density of $1 \times 10^{8} \mathrm{~A} / \mathrm{cm}^{2}$ and a varied polarization of the conduction electrons $P$ from 0.2 to 0.5 . The numerically calculated results of $\cos \theta$ and frequency are shown in Figure 4. Similar to the spin current, the increase in the polarization of conduction electrons results in a decrease in the oscillation angle and an increase in the oscillation frequency because more spin torque is injected to the oscillation layer.

3.3. Anisotropy Energy Density. The simulation parameters are the same as those in Section 3.1, except for a fixed current density of $1 \times 10^{8} \mathrm{~A} / \mathrm{cm}^{2}$ and a varied anisotropy 

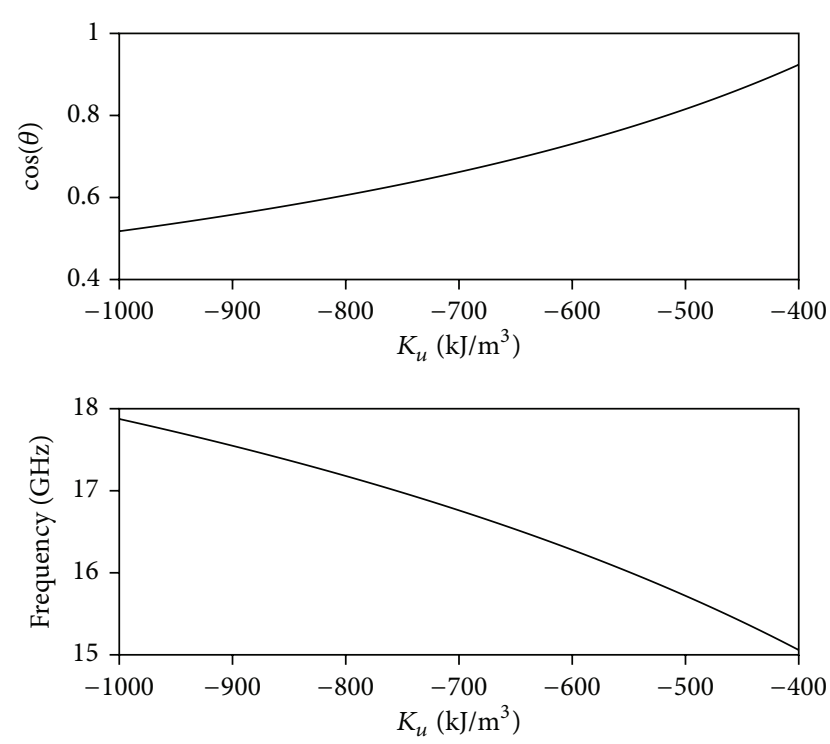

FIGURE 5: Effects of anisotropy energy density on STO.

energy density $K_{u}$ of the oscillation layer from $-1000 \mathrm{~kJ} / \mathrm{m}^{3}$ to $-400 \mathrm{~kJ} / \mathrm{m}^{3}$. A high negative anisotropy energy density (absolute value) results in a high oscillation angle (low $\cos \theta$ ) and a high value of the spin transfer efficiency function $G(\theta)$; thus the oscillation frequency increases with the increase of the anisotropy energy density (absolute value) as shown in the numerically calculated results in Figure 5.

3.4. Saturation Magnetization. The simulation parameters are the same as those in Section 3.1, except for a fixed current density of $1 \times 10^{8} \mathrm{~A} / \mathrm{cm}^{2}$ and a varied saturation magnetization $M_{0}$ from $400 \mathrm{kA} / \mathrm{m}$ to $800 \mathrm{kA} / \mathrm{m}$. The high saturation magnetization results in a low magnetic anisotropy field and a high demagnetizing field, which result in a low oscillation angle (high $\cos \theta$ ) and a low value of the spin transfer efficiency function $G(\theta)$. Besides the $G(\theta)$, the STO frequency is inversely proportional to the $M_{s}$ as shown in (7a); thus the oscillation frequency decreases with the increase of the saturation magnetization as shown in the numerically calculated results in Figure 6.

3.5. Total Applied Magnetic Field. The simulation parameters are the same as those in Section 3.1, except for a fixed current density of $1 \times 10^{8} \mathrm{~A} / \mathrm{cm}^{2}$ and a varied total applied magnetic field $H_{a z}$ in $+z$ direction from the 0 to 10000 Oe. The high $H_{a z}$ results in a low oscillation angle and a low value of the spin transfer efficiency function $G(\theta)$. Therefore, the oscillation frequency decreases with the increase of the $H_{a z}$ as shown in the numerically calculated results in Figure 7.

\section{Conclusions}

Using modified LLG equation, we derived formulas to solve the oscillation frequency and oscillation angle for the switchable spin torque oscillator (STO) with negative magnetic
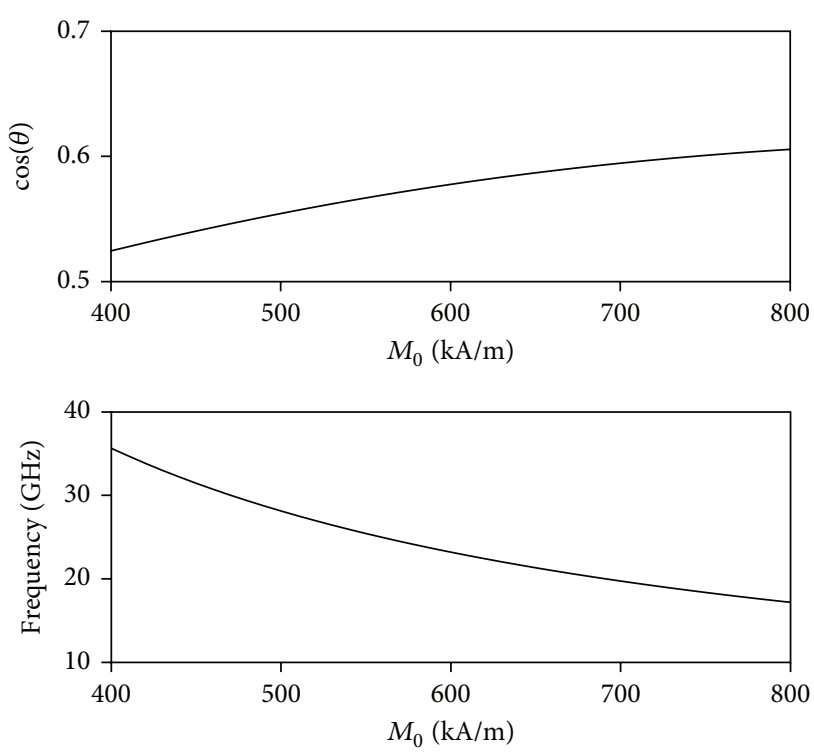

FIGURE 6: Effects of saturation magnetization on STO.
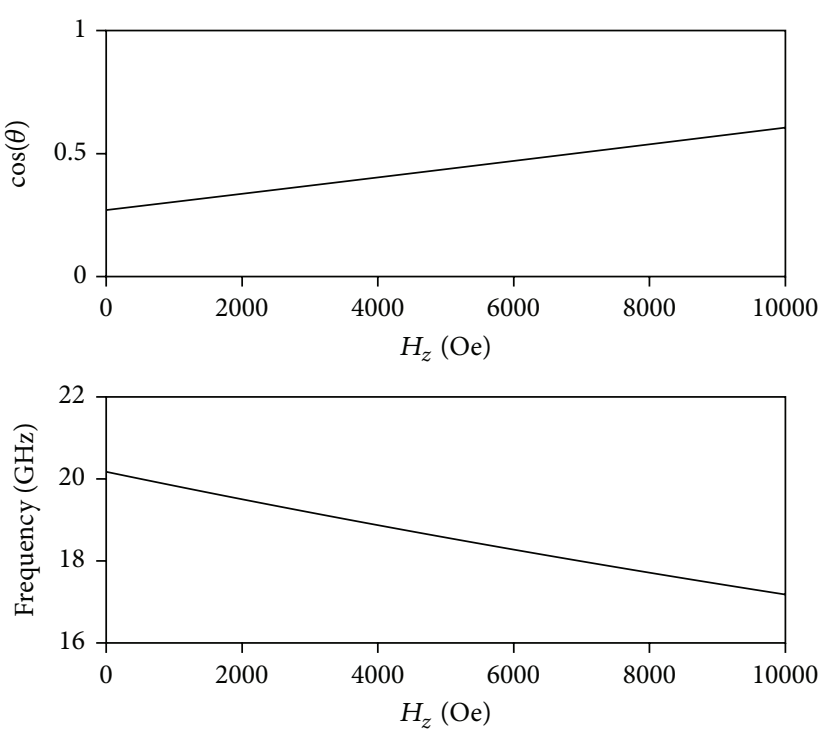

FIGURE 7: Effects of applied magnetic field on STO.

anisotropy oscillation layer. The STO keeps the same oscillation frequency, while its oscillation direction reverses after the flip of applied external field in the $z$ direction. The oscillation angle increases with the increase of the negative anisotropy energy density (absolute value) but decreases with the increase of spin current, the polarization of conduction electrons, the saturation magnetization, and the total applied magnetic field in the $z$ direction. The STO frequency increases with the increase of spin current, the polarization of conduction electrons, and the negative anisotropy energy density (absolute value) but decreases with the increase of the saturation magnetization and the total applied magnetic field in the $z$ direction. The findings in this paper offer guidelines 
for STO design for microwave assisted magnetic recording at ultrahigh density.

\section{Conflict of Interests}

The authors declare that there is no conflict of interests regarding the publication of this paper.

\section{References}

[1] J. C. Slonczewski, "Current-driven excitation of magnetic multilayers," Journal of Magnetism and Magnetic Materials, vol. 159, no. 1-2, pp. L1-L7, 1996.

[2] M. D. Stiles and J. Miltat, "Spin-transfer torque and dynamics," in Spin Dynamics in Confined Magnetic Structures III, pp. 225308, Springer, Berlin, Germany, 2006.

[3] Z. Li and S. Zhang, "Magnetization dynamics with a spintransfer torque," Physical Review B-Condensed Matter and Materials Physics, vol. 68, no. 2, Article ID 024404, 2003.

[4] J.-G. Zhu, X. Zhu, and Y. Tang, "Microwave assisted magnetic recording," IEEE Transactions on Magnetics, vol. 44, no. 1, pp. 125-131, 2008.

[5] J.-G. Zhu and Y. Wang, "Microwave assisted magnetic recording utilizing perpendicular spin torque oscillator with switchable perpendicular electrodes," IEEE Transactions on Magnetics, vol. 46, no. 3, pp. 751-757, 2010.

[6] K. Yoshida, M. Yokoe, Y. Ishikawa, and Y. Kanai, "Spin torque oscillator with negative magnetic anisotropy materials for MAMR," IEEE Transactions on Magnetics, vol. 46, no. 6, pp. 2466-2469, 2010.

[7] J. S. Moodera and G. Mathon, "Spin polarized tunneling in ferromagnetic junctions," Journal of Magnetism and Magnetic Materials, vol. 200, no. 1, pp. 248-273, 1999. 

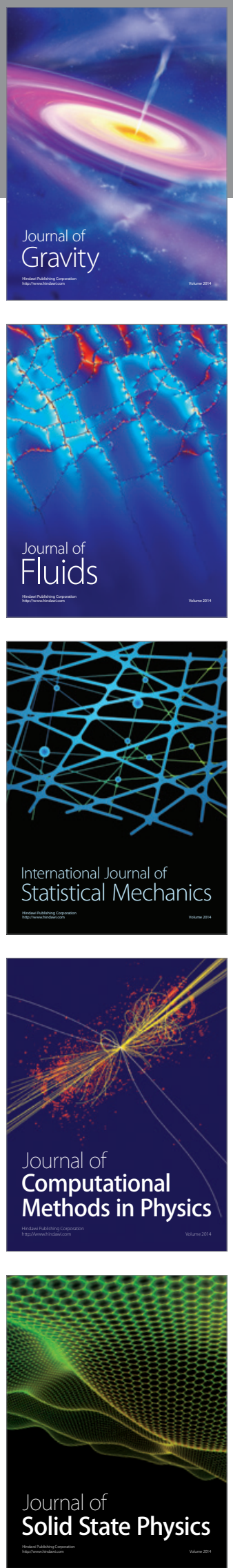

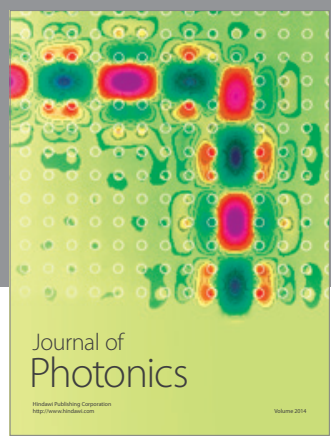

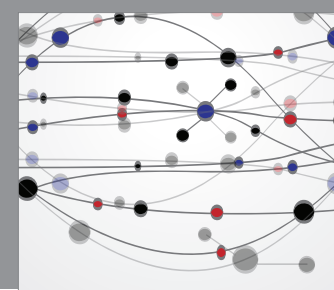

The Scientific World Journal

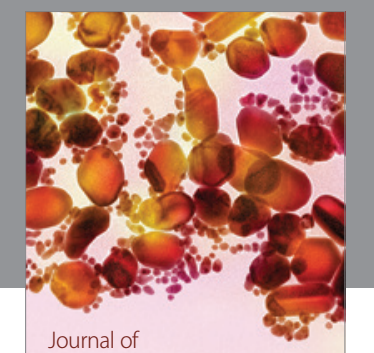

Soft Matter
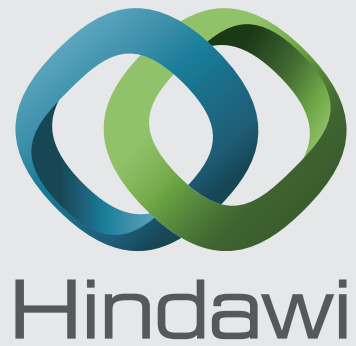

Submit your manuscripts at

http://www.hindawi.com
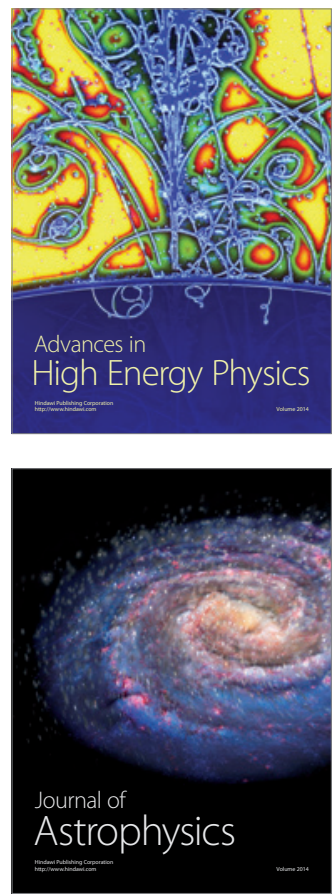
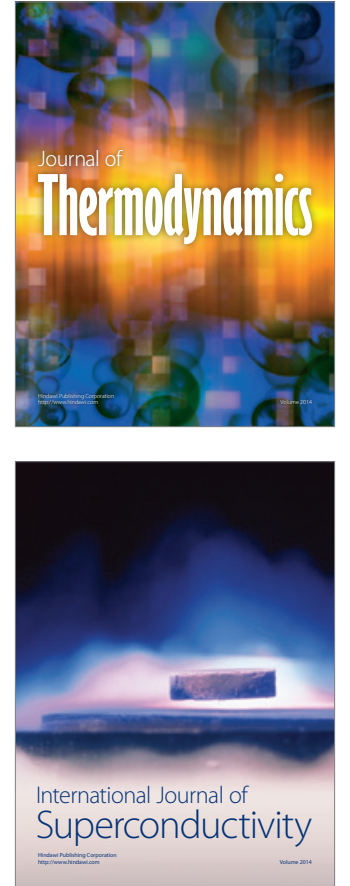
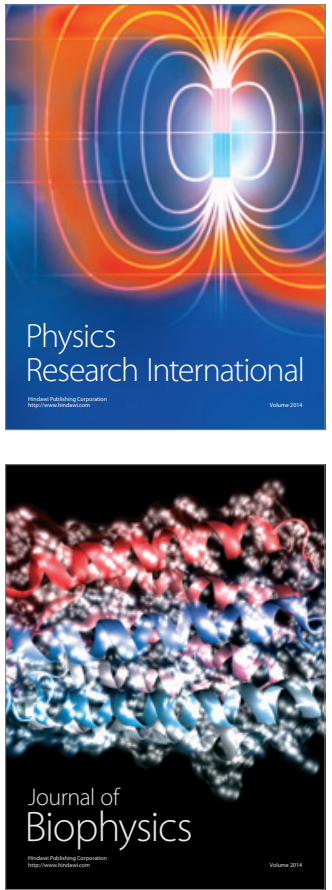
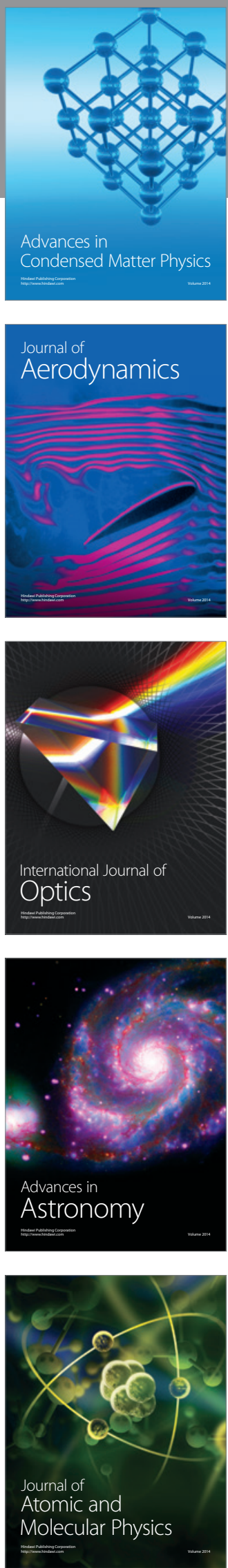